\title{
Systemic inflammatory activation in patients with acute coronary syndrome secondary to nonatherosclerotic spontaneous coronary artery dissection
}

\author{
Yigit Canga, ${ }^{1}$ Tolga Sinan Guvenc, ${ }^{1}$ Ali Nazmi Calik, ${ }^{1}$ Mehmet Baran Karatas, ${ }^{1}$ Tahir Bezgin, ${ }^{2}$ \\ Tolga Onuk, ${ }^{1}$ Ahmet Okan Uzun, ${ }^{1}$ Veysel Ozan Tanik, ${ }^{1}$ Baris Gungor, ${ }^{1}$ Osman Bolca ${ }^{1}$ \\ ${ }^{1}$ Department of Cardiology, Siyami Ersek Cardiovascular and Thoracic Surgery Center, Istanbul, Turkey \\ ${ }^{2}$ Department of Cardiology, Gebze Fatih State Hospital, Izmit, Turkey
}

\begin{abstract}
OBJECTIVE: Pathological studies have suggested that local inflammation, particularly eosinophilic infiltration of the adventitia, could be related to nonatherosclerotic spontaneous coronary artery dissection (NA-SCAD). However, the role of systemic inflammation in the pathogenesis of NA-SCAD remains unknown. Our aim was to investigate systemic inflammatory activation in patients with an acute coronary syndrome (ACS) secondary to NA-SCAD.
\end{abstract}

METHODS: The institutional electronic medical database was reviewed, and 22 patients with NA-SCAD-ACS were identified after the review. Furthermore, 30 random patients with CAD-ACS and 30 random subjects without any history of CAD or ACS with demographic and clinical characteristics similar to those of NA-SCAD-ACS patients were identified from the institutional database to be included in the study.

RESULTS: Patients with NA-SCAD-ACS and those with CAD-ACS both had higher white blood cell and neutrophil counts than controls. Neutrophil-lymphocyte ratio (NLR) and C-reactive protein (CRP) levels were only significantly higher in the NA-SCADACS group [2.01 (1.54-6.17) for NLR and $0.70(0.13-2.70)$ for CRP] than in the controls [1.55 (1.27-2.13), $p=0.03$ for NLR and 0.15 (0.10-0.43), $\mathrm{p}=0.049$ for CRP]; however, there were no differences between the NA-SCAD-ACS and CAD-ACS groups [1.91 (1.41-2.78) for NLR and 0.41 (0.09-1.10) for CRP, $\mathrm{p}>0.05$ for both comparisons] regarding all tested parameters.

CONCLUSION: The degree of inflammatory activation in NA-SCAD-ACS patients was similar to, or even greater than, that in CAD-ACS patients; thus, suggesting a role of inflammation in the pathophysiology of NA-SCAD-ACS.

Keywords: Acute coronary syndrome; inflammation; spontaneous coronary artery dissection.

Cite this article as: Canga Y, Güvenc TS, Calik AN, Karatas MB, Bezgin T, Onuk T, et al. Systemic inflammatory activation in patients with acute coronary syndrome secondary to nonatherosclerotic spontaneous coronary artery dissection. North Clin Istanb 2018;5(3):186-194.

Spontaneous coronary artery dissection (SCAD) is a $\checkmark$ rare coronary pathology that is caused by a non-provoked separation of the medial and adventitial layers of the coronary artery secondary to an intimal tear or hemorrhage within the vessel wall. While SCAD could affect both atherosclerotic and non-atherosclerotic coronary arteries, atherosclerotic SCAD is generally considered a variant of atherosclerotic coronary artery disease (CAD) as the dissection is related to the rupture of the atheroma and is limited to a short segment of the coronary artery as the progression is impeded by medial scarring secondary to CAD [1]. Nonatherosclerotic SCAD (NA-

Received: June 06, 2017 Accepted: September 20, 2017 Online: May 24, 2018

Correspondence: Dr. Yigit CANGA. Siyami Ersek Gogus Kalp ve Damar Cerrahisi Egitim ve Arastirma Hastanesi, Kardiyoloji Bolumu, Istanbul, Turkey.

Phone: +902164592766 e-mail: canga81@hotmail.com

(c) Copyright 2018 by Istanbul Provincial Directorate of Health - Available online at www.northclinist.com 
SCAD) comprises $0.1 \%-0.28 \%$ of all angiographicallyproven myocardial infarctions [2] and is more frequently observed in women, especially during peripartum period but may also affect older women [3].

Several autopsy reports and studies have associated inflammation, especially eosinophilic infiltration of the outer media and adventitia of the coronary vessel, with the occurrence of NA-SCAD $[4,5]$. A noteworthy similarity exists between the eosinophilic coronary periarteritis (ECPA) and NA-SCAD, as both conditions are characterized by eosinophilic infiltration with mast cell degranulation in relatively short segments of the coronary arteries [6]. Other manifestations of systemic allergy or inflammation, such as asthma, may or may not accompany NA-SCAD and ECPA, but eosinophilia or widespread arteritis in systemic vessels is usually missing, suggesting that these phenomena may represent a standalone disorder $[6,7]$. Besides these observations, however, an evaluation of hematologic or circulatory markers of inflammation was not attempted in patients with NASCAD. Systemic inflammation is a well-known feature of atherosclerotic CAD as inflammation plays a crucial role in the evolution of atheroma from the initial "fatty streak" lesions to complex atherosclerotic plaques responsible for acute coronary events [8].

We hypothesized that systemic inflammation may also play a role in NA-SCAD due to the association of this condition with periarterial inflammation. In this retrospective analysis, we aimed to investigate the presence of systemic inflammatory markers in NA-SCAD patients with a diagnosis of acute coronary syndrome (NA-SCAD-ACS) and compare our findings with age- and sex-matched subjects with ACS secondary to atherosclerotic coronary disease (CAD-ACS) and healthy individuals. As a secondary aim, we investigated the angiographic characteristics, management strategies, and in-hospital outcomes in NA-SCAD-ACS and CAD-ACS patients.

\section{MATERIALS AND METHODS}

All coronary angiographies performed between 2011 and 2015 and stored in the institutional database were reviewed for angiography reports containing the words "coronary" and "dissection." Among 30255 coronary angiography reports, 187 met the criteria. Stored angiographic images for these reports were then evaluated by two cardiologists experienced in invasive procedures (YÇ and $\mathrm{MBK}$ ). NA-SCAD was defined as an intraluminal filling defect during contrast injection or persistent staining of the artery following contrast injection that was not interpreted as an obstructive coronary atheroma or intracoronary thrombus, and the artery in which the dissection was observed should be free from any coronary atherosclerotic plaque. To be eligible for inclusion in the study, dissection should be evident in at least two angiographic projections. In case of disagreement between two investigators, a third investigator (TSG) evaluated the angiogram in question and decided whether the patient would be included in the analysis. Patients in whom the intimal tear was in close proximity to the catheter tip were excluded due to the possibility of iatrogenic dissection. Other exclusion criteria included known inflammatory diseases, an acute infection at the time of angiography, known hematologic disorders that affect white blood cells, known malignancy, prior splenectomy, and additional exclusion criteria specified in the "laboratory analysis" section. While age was not an exclusion criterion, none of the patients were aged under 18 years as these patients were not accepted in the adult cardiology unit. Finally, patients diagnosed with NA-SCAD under non-emergent conditions (i.e., an evaluation for stable angina) were excluded due to the possibility of different inflammatory conditions in stable patients. Of the initial 187 patients, 22 met the inclusion criteria and were included in the study (Fig. 1). In addition to the patients with NA-SCAD, records for two additional

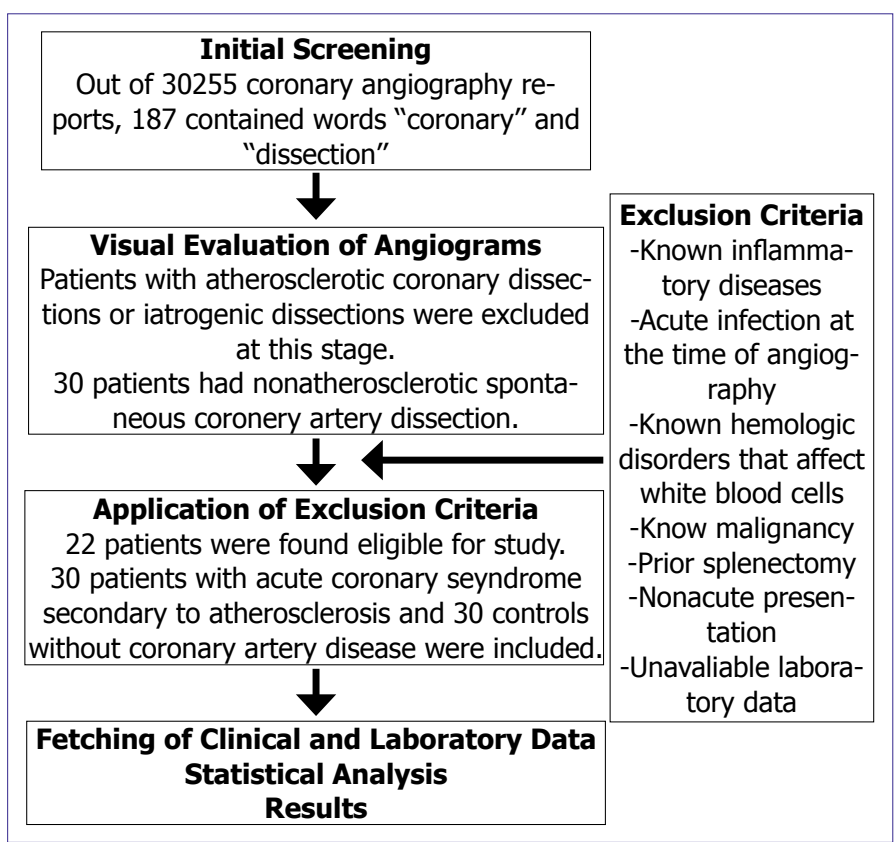

FIGURE 1. Flow diagram of the study. 
groups of subjects were collected to compare the degree of inflammation in patients with NA-SCAD. In the first group, records of 30 age- and gender-matched patients who were hospitalized in the study institution and who underwent coronary angiography for ACS [either STsegment elevation myocardial infarction (STEMI) or unstable angina/non-ST-segment elevation myocardial infarction (UA/NSTEMI)] and were diagnosed with CAD-ACS were collected to form CAD-ACS group. Records of a second set of 30 age- and sex-matched subjects without a cardiovascular disease who were admitted to the study institution for a routine check-up were collected to serve as controls. Subjects in the CAD-ACS and control groups were randomly selected using computer-generated numbers, and aforementioned exclusion criteria were also applied to the CAD-ACS and control groups.

All the records were retrieved using institutional electronic medical database (EMD). In addition to the angiographic records, patients' demographic characteristics, prior medical history, laboratory values, and data on inhospital outcomes were retrieved from EMD.

This analysis was performed according to the principles of the Declaration of Helsinki, and a local Ethics Committee had approved the study.

\section{Laboratory analysis}

Per institutional protocol, blood is withdrawn for complete blood count and biochemical analysis from all patients who undergo coronary angiography. The blood is withdrawn and studied one day before coronary angiography for patients undergoing elective angiography. For patients undergoing emergent angiography, the blood is withdrawn immediately before angiography. For this analysis, complete blood count and $\mathrm{C}$-reactive protein (CRP) values that preceded angiography were used if the blood was withdrawn within $24 \mathrm{~h}$ before angiography. Laboratory analyses performed using blood withdrawn $>24$ h before angiography or blood withdrawn following the study were not used. Laboratory records were not used if the laboratory had indicated possible hemolysis of the sample, and the patient was excluded from the study if there were no other laboratory analyses that preceded angiography. For healthy controls, blood that was analyzed during check-up was used for statistical analyses.

As an institutional protocol, blood withdrawn from the patients was collected into an EDTA - containing tube for complete blood count evaluation and into a dry tube for biochemical analysis. All the samples collected for analysis were studied within half an hour of the blood withdrawal. An automatic Coulter analyzer was used for evaluating complete blood counts (Beckman Coulter LH 750, Fullerton, CA). CRP levels were determined using a BN II model Nephelometer (Dade Behring, Marburg, Germany).

\section{Statistical analysis}

All statistical analyses were performed using SPSS 17.0 statistical analysis software (IBM Inc, USA). Continuous variables were presented as mean $\pm \mathrm{SD}$ if distributed normally, while median and interquartile ranges were provided in case of a heterogenous distribution. Categorical variables were provided as percentages; KolmogorovSmirnov and Levene tests were used to test for normal distribution and homogeneity of data, respectively. A one-way ANOVA was preferred for parameters with a homogenous distribution, and post-hoc analyses were performed with Tukey's HSD. For parameters with a heterogenous distribution or in case of an inequality of variances, Kruskal-Wallis test was used; Mann-Whitney U test was used for analysis of subgroups. For categorical variables, $\chi^{2}$ test or Fisher's exact test was preferred according to the observed and expected cell counts. A univariate analysis was performed to determine significant predictors for SCAD. For all comparisons, a $\mathrm{p}$ value of $<0.05$ was accepted as the cut-off value for significance.

\section{RESULTS}

Demographic, clinical, and laboratory findings are presented in Table 1 . There were no differences between the groups regarding age, gender, or risk factors for atherosclerotic CAD. Both the NA-SCAD-ACS and CAD-ACS groups had higher absolute total leucocyte and neutrophil counts than the control group. In contrast, both neutrophil-lymphocyte ratio (NLR) and CRP levels were significantly higher in the NA-SCADACS group than in the control group $(p=0.009$ and $p=0.007$, respectively); however, both NLR and CRP levels were not different between the CAD-ACS and control groups $(\mathrm{p}=0.07$ and $\mathrm{p}=0.24$, respectively) (Fig. 2). Neither the absolute eosinophil count nor the lymphocyte count differed between the groups, yet the percentages of eosinophils and lymphocytes were signifcantly lower in the NA-SCAD-ACS group than in the control group ( $p=0.048$ and $p=0.027$, respectively). No significant differences were observed between the CAD- 
TABLE 1. Summary of demographic, clinical and laboratory variables between study groups

\begin{tabular}{|c|c|c|c|c|}
\hline Parameter & NASCAD-ACS $(n=22)$ & CAD-ACS $(n=30)$ & Control $(n=30)$ & $\mathrm{P}$ \\
\hline \multicolumn{5}{|c|}{ Demographic and Clinical Variables } \\
\hline Age $(y)$ & $47.0 \pm 12.3$ & $48.5 \pm 0.1$ & $45.6 \pm 9.1$ & 0.53 \\
\hline Gender (Female) n (\%) & 50 & 40 & 40 & 0.72 \\
\hline Hypertension n (\%) & 25 & 36 & 30 & 0.85 \\
\hline Diabetes n (\%) & 16 & 17 & 13 & 0.91 \\
\hline Hyperlipidemia n (\%) & 5 & 17 & 6 & 0.27 \\
\hline Smoking n (\%) & 32 & 40 & 17 & 0.13 \\
\hline \multicolumn{5}{|l|}{ Laboratory Variables } \\
\hline WBC $\left(10^{3} / \mathrm{mm}^{3}\right)$ & $9.68 \pm 3.44 \dagger$ & $9.39 \pm 2.80 \dagger$ & $6.91 \pm 1.70$ & $<0.001$ \\
\hline $\operatorname{PNL}\left(10^{3} / \mathrm{mm}^{3}\right)$ & $6.55 \pm 3.30 \dagger$ & $5.79 \pm 2.73+$ & $3.81 \pm 1.28$ & $<0.001$ \\
\hline PNL (\%WBC) & $63.61 \pm 14.55 \dagger$ & $59.99 \pm 11.53$ & $54.45 \pm 8.59$ & 0.02 \\
\hline Eosinophile $\left(10^{3} / \mathrm{mm}^{3}\right)$ & $0.15 \pm 0.12$ & $0.17 \pm 0.13$ & $0.19 \pm 0.12$ & 0.52 \\
\hline Eosinophile (WBC\%) & $1.76 \pm 1.50 \dagger$ & $1.86 \pm 1.31$ & $2.71 \pm 1.46$ & 0.03 \\
\hline Lymphocyte $\left(10^{3} / \mathrm{mm}^{3}\right)$ & $2.18 \pm 0.79$ & $2.71 \pm 0.99$ & $2.36 \pm 0.65$ & 0.07 \\
\hline Lymphocyte (WBC\%) & $26.77 \pm 14.25 \dagger$ & $30.28 \pm 10.39$ & $34.70 \pm 7.56$ & 0.03 \\
\hline NLR & $2.01(1.54-6.17)+$ & $1.91(1.41-2.78)$ & $1.55(1.27-2.13)$ & 0.03 \\
\hline ELR & $0.07 \pm 0.05$ & $0.06 \pm 0.05$ & $0.08 \pm 0.04$ & 0.32 \\
\hline C-Reactive Protein (mg/dl) & $0.70(0.13-2.70)+$ & $0.41(0.09-1.10)$ & $0.15(0.10-0.43)$ & 0.049 \\
\hline
\end{tabular}

WBC; White blood cell, PNL; polymorphonuclear leucocytes, NLR, neutrophil-to-lymphocyte ratio, ELR; eosinophile-to-lymphocyte ratio; †: Significantly different at $\mathrm{p}<0.05$ level as compared to control group.

ACS and control groups regarding the latter parameters. For all the comparisons presented in Table 1, no significant differences were found between the NA-SCADACS and CAD-ACS groups.

Table 2 summarizes the angiographic characteristics, in-hospital management, and outcomes in the NASCAD-ACS and CAD-ACS groups. Multivessel disease and left circumflex lesions were less frequent in the NA-SCAD-ACS group, but the length of the dissected segments was longer than that of the atherosclerotic lesions, thus, necessitating longer stents if percutaneous intervention was chosen for management. However, a conservative strategy was much more common in the NA-SCAD-ACS group compared with that in the CADACS group. All patients managed with an initial conservative strategy in the NA-SCAD-ACS group presented with unstable angina or non-ST elevation MI as opposed to STEMI (44\% vs. $0 \%$ ). For both the NA-SCAD-ACS and CAD-ACS groups, the in-hospital mortality rate was $0 \%$, with only one patient in each group experiencing a MACE. While an initial conservative strategy was the more common management strategy in the NA-SCADACS group, the frequency of in-hospital major cardio- vascular events did not differ between the groups; however, the ejection fraction in the NA-SCAD-ACS group was significantly lower than that in the CAD-ACS group (Table 2). Finally, major cardiovascular events were similar in NA-SCAD-ACS groups managed conservatively or invasively ( $0 \%$ in the conservative group vs. $12.5 \%$ in the early invasive group, $\mathrm{p}=0.36$ ), and there were no differences in the ejection fraction between these groups before discharge (49.28 $\pm 12.69 \%$ in the conservative group vs. $48.75 \pm 11.57 \%$ in the early invasive group, $p=0.87$ ).

On univariate analysis, none of the demographic, clinical, or laboratory parameters appeared to have sufficient discriminative capacity to differentiate the NA-SCADACS group from the CAD-ACS group; thus, a multivariate analysis was not attempted.

\section{DISCUSSION}

The major findings of the present study can be summarized as follows: i) similar to CAD-ACS patients, systemic inflammatory markers were increased in NASCAD-ACS patients; ii) while CRP levels and NLR were higher in the NA-SCAD-ACS group, there were no 

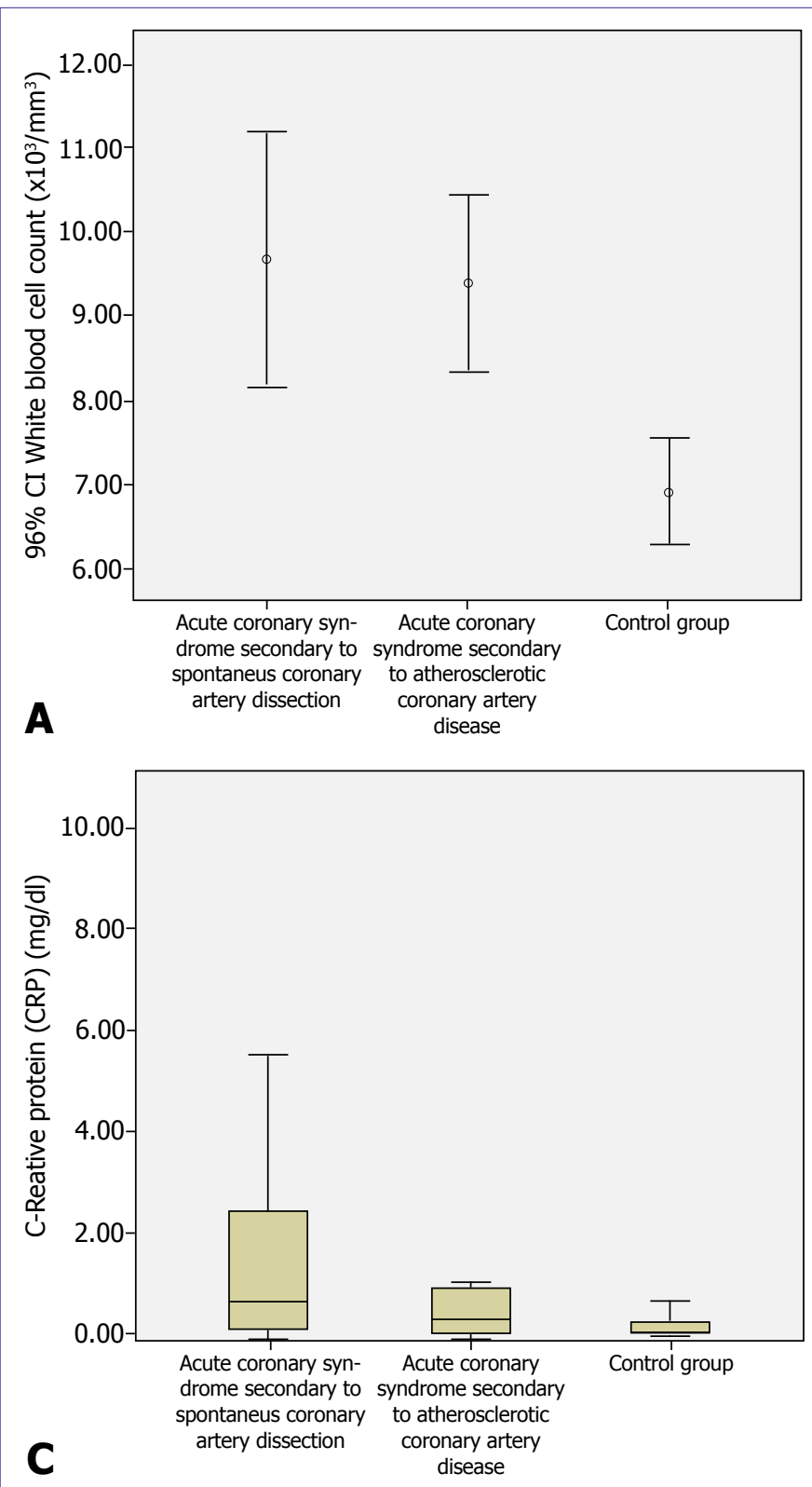

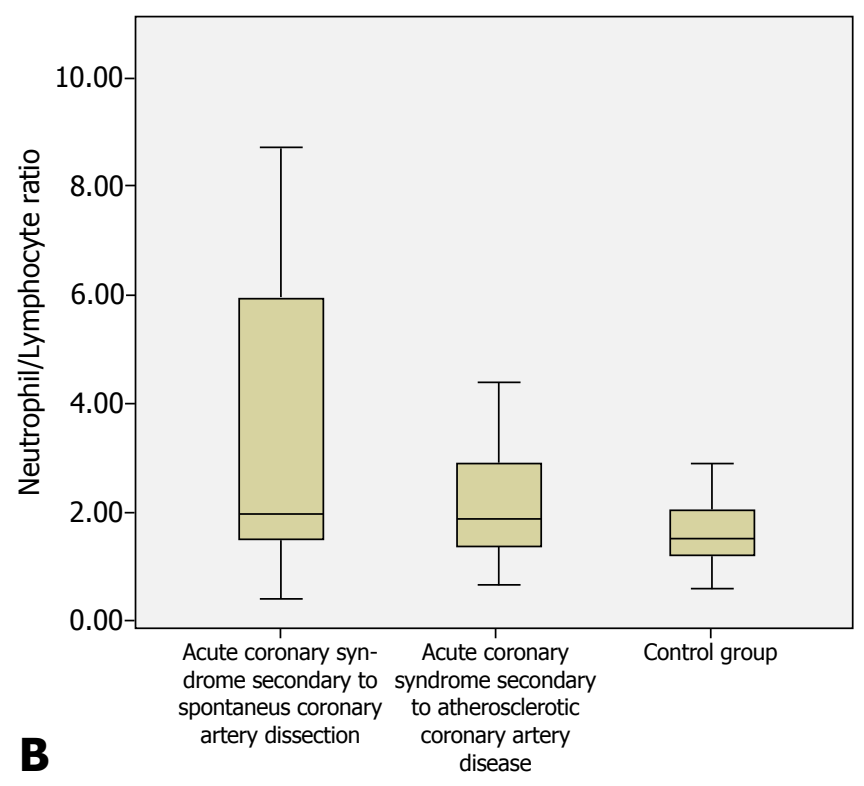

FIGURE 2. White blood cell count (A), neutrophil-lymphocyte ratio (NLR) (B) and C-reactive protein (CRP) levels (C) among the study groups. Patients with an ACS secondary to spontaneous coronary artery dissection (NA-SCAD-ACS) had significantly higher white blood cell counts, NLR, and CRP levels as than the controls, whereas only total white blood cell count was significantly higher in patients with an acute coronary syndrome secondary to atherosclerotic coronary artery disease (CAD-ACS). None of the parameters, however, differed between the NA-SCAD-ACS and CAD-ACS groups. See text for details. significant differences between the NA-SCAD-ACS and CAD-ACS groups regarding inflammatory biomarkers; iii) the percentage of eosinophils in the peripheral blood was decreased in NA-SCAD-ACS patients; and iv) a conservative approach was more frequently preferred in NA-SCAD-ACS patients, and this management strategy does not appear to increase in-hospital events. This last observation, however, should be interpreted with extreme caution due to the low sample size and retrospective nature of the study.

Inflammation is a major pathophysiologic factor that affects all phases of atherosclerotic CAD [9]. Subjects with elevated levels of inflammatory biomarkers, including CRP, serum amyloid-associated protein, or inter- leukin-6 were at risk for future ischemic events [10-14]. Following ST - and non-ST elevation myocardial infarction, several inflammatory biomarkers including (but not limited to) CRP levels, total white blood cell count, and NLR were associated with the risk of mortality and future cardiovascular events [15-21]. It is postulated that inflammation modulates the tendency of an atherosclerotic lesion to rupture, thus, increasing the vulnerability of an individual plaque [22]. A similar pathophysiologic mechanism was proposed for acute arterial dissection, where inflammation was hypothesized to increase the risk for intimal tears [23-26]. The role of inflammation in patients with other types of arterial dissection, such as SCAD, is less clear. Present findings showed that the 
TABLE 2. Angiographic characteristics, in hospital management strategies and in-hospital outcomes for study groups.

\begin{tabular}{|c|c|c|c|}
\hline Parameter & NASCAD-ACS $(n=22)$ & CAD-ACS $(n=30)$ & $P$ \\
\hline \multicolumn{4}{|l|}{ Lesion Characteristics } \\
\hline Presentation (STEMI) n (\%) & 18.2 & 20 & 1.0 \\
\hline Presentation (UA/NSTEMI) n (\%) & 81.8 & 80 & 1.0 \\
\hline Involvement of LMCA n (\%) & 13.6 & 3.3 & 0.17 \\
\hline Involvement of LAD n (\%) & 40.9 & 63.3 & 0.10 \\
\hline Involvement of LCx $\mathrm{n}(\%)$ & 9.1 & 50 & 0.002 \\
\hline Involvement of RCA n (\%) & 59.1 & 46.7 & 0.38 \\
\hline Multivessel Disease n (\%) & 13.6 & 40 & 0.04 \\
\hline Reference Diameter (mm) & $2.79 \pm 0.47$ & $2.83 \pm 0.37$ & 0.71 \\
\hline Lesion Length (mm) & $26.5 \pm 18.7$ & $17.1 \pm 7.1$ & 0.03 \\
\hline \multicolumn{4}{|l|}{ In-Hospital Management } \\
\hline Conservative $\mathrm{n}(\%)$ & 36.4 & 6.7 & 0.01 \\
\hline Percutaneous Intervention $\mathrm{n}(\%)$ & 31.8 & 73.3 & 0.003 \\
\hline CABG $n(\%)$ & 31.8 & 13.3 & 0.11 \\
\hline \multicolumn{4}{|l|}{ In-Hospital Follow-Up } \\
\hline Predischarge EF n (\%) & $49.1 \pm 12.0$ & $56.0 \pm 7.9$ & 0.04 \\
\hline In-Hospital Mortality n (\%) & 0 & 0 & $\mathrm{~N} / \mathrm{A}$ \\
\hline In-Hospital MACE n (\%) & 4.5 & 3.3 & 1.0 \\
\hline
\end{tabular}

STEMI; ST-elevation myocardial infarction, UA/NSTEMI; unstable angına or non-ST elevation myocardial infarction, LMCA; left main coronary artery, LAD; left anterior descending artery, LCX; left circumflex artery, RCA; right coronary artery, CABG; coronary artery bypass grafting, EF; ejection fraction, MACE; major cardiovascular adverse effects.

degree of inflammation in NA-SCAD-ACS was similar to that in CAD-ACS, as no significant differences were found between these two conditions regarding the inflammatory biomarkers. While this preliminary study suggests that inflammation is present in NA-SCADACS, the pathophysiologic and clinical relevance of inflammation in NA-SCAD-ACS remains obscure, thus, necessitating further studies to better understand the role of inflammation in NA-SCAD-ACS.

As mentioned before, previous studies have found that virtually all biomarkers of inflammation, including CRP levels and NLR, are elevated in CAD-ACS patients, and the magnitude of inflammation is associated with the outcomes in CAD-ACS. In the present study, we found that both CRP levels and NLR were higher in the CAD-ACS group than in the controls; however, the difference was not significant. As the levels of other biomarkers of inflammation were significantly higher in the CAD-ACS group (Table 1), we assume that the cause of this latter finding is the low statistical power of the study rather than a true lack of difference between the groups. It is worth noting that the mean NLR and
CRP levels in the NA-SCAD-ACS group were higher than those in the CAD-ACS group, although this finding did not reach significance. Whether this difference represents a higher degree of inflammatory reaction in NA-SCAD-ACS patients or simply represents a random effect could not be inferred from the present study due to its low statistical power and retrospective design. In our opinion, this latter observation deserves further research as a better understanding of the role of inflammation in NA-SCAD-ACS might have therapeutic value.

A further concern regarding the inflammation in patients with NA-SCAD is the location and type of inflammatory reaction in the affected vessel. Several case studies have reported periarterial inflammation, most notably eosinophilic infiltration, as a postmortem finding in patients with NA-SCAD $[6,27]$. It has been hypothesized that the proteolytic enzymes originating from eosinophils cleave collagen and elastin within the tunica media, thus, weakening the vessel wall and facilitating coronary dissection [28]. However, it is unknown whether these findings represent a cause-and-effect relationship or merely a reactive phenomenon secondary to 
dissection $[4,29]$. Pathologic findings in patients with NA-SCAD share several similarities with ECPA, which is postulated as a specific type of monoarteritis confined to coronary arteritis [30]. Our findings imply that eosinophilia is not a common condition in patients with NA-SCAD (Table 1). Hypereosinophilia increases the tendency for eosinophilic involvement in several organs including skin, lung, or the myocardium, but hypereosinophilia is not a prerequisite for isolated eosinophilic inflammation in individual tissues. Considering the findings of the present study and those of previous autopsy studies together, it can be suggested that coronary involvement in NA-SCAD is not caused by hypereosionphilia, but it is rather a result of an organ-specific disease. The importance of relative eosinopenia observed in NA-SCAD-ACS patients is unclear because the absolute eosinophil count was similar among the groups and there was a similar tendency for relative eosinopenia in the CAD-ACS group. These findings suggest that the cause of relative (but not absolute) eosinopenia could be an increase in the absolute neutrophil count secondary to ACS, thus, reducing the percentage of eosinophils in circulation.

Randomized controlled trials performed within the last 20 years have proved that an immediate reperfusion strategy for STEMI and an early invasive strategy for US/NSTEMI reduces mortality and improves outcomes. Such strategies, however, were tested in patients with atherothrombotic lesions and should not be necessarily generalized to patients with other types of acute coronary events. Several studies have, in fact, reported that outcomes of a conservative strategy in NA-SCADACS are non-inferior to those of an early invasive strategy $[3,31]$. Present findings suggest that in-hospital mortality or major cardiovascular events in NA-SCADACS patients were similar to those of an age- and gendermatched sample of patients with CAD-ACS, although an early reperfusion strategy was not considered in $63 \%$ of the patients with NA-SCAD. However, ejection fraction before discharge was lower in the NA-SCADACS group, which may increase the overall risk for adverse events in patients with NA-SCAD-ACS when the strong prognostic impact of left ventricular contractility on long-term survival is considered [32]. As in-hospital cardiovascular adverse effects and predischarge ejection fraction were similar between patients managed conservatively and those managed with an invasive strategy, it is unclear why patients with NA-SCAD-ACS had a lower predischarge ejection fraction than CAD-ACS patients.
Finally, it should be emphasized that the present study is grossly underpowered to detect the differences in outcomes or mortality, and the retrospective nature of the study prevents making healthy comparisons between the two management strategies. Therefore, our results should be interpreted with extreme caution as there are no definitive randomized controlled trials that compare an early invasive vs. conservative strategy in patients with NA-SCAD-ACS. These latter results should not be generalized to NA-SCAD-ACS patients with STEMI as all patients with STEMI were managed with immediate reperfusion strategy rather than conservative management.

As the management strategies may differ for NASCAD-ACS and CAD-ACS patients, distinguishing between these two conditions prior to obtaining an angiogram could aid management decisions. Previous studies have indicated that NA-SCAD patients are younger and predominantly females, although this latter finding is controversial as recent studies have demonstrated that NA-SCAD could be frequently present in males as well [31]. As these patients were younger, it is considered that risk factors for CAD are less frequently present in patients with NA-SCAD, although a control group was not used and the findings were based on individual case series [33]. Compared with an age- and gender-matched CAD-ACS population, however, we did not find any differences regarding the frequency of conventional coronary risk factors in NA-SCAD-ACS patients, suggesting that conventional risk factors were inadequate to discriminate between these two conditions. Similarly, inflammatory markers including total leucocyte count, neutrophil or eosinophil count, NLR, and CRP levels had an inadequate discriminatory capacity for distinguishing NA-SCAD-ACS from CAD-ACS. As the primary aim of the present study was to assess the presence of inflammation and these analyses were secondary, the reported findings should be regarded as preliminary and not definitive.

\section{Study limitations}

The present study is a retrospective analysis of a small number of NA-SCAD-ACS cases obtained from the database of a single study center. A major limitation of this study is the lack of data on intracoronary imaging studies such as intravascular ultrasound or optical coherence tomography, as these studies were not routinely performed in all the patients. Invasive coronary angiography is an inadequate tool for the diagnosis of intra- 
coronary dissections, and only angiographically visible (type I) coronary dissections could be diagnosed using angiographic data. Therefore, our results should not be generalized to all coronary dissections. A conventional method was used for CRP level measurements in the study period; thus, the findings could be different if the analyses were repeated using a high-sensitivity CRP kit. As mentioned before, the sample size of the study is severely limited to make assumptions on outcomes or management strategies, and the retrospective nature of the study prevents making direct comparisons between conservative and early invasive strategies. Furthermore, the results have indicated that there was a tendency toward more frequent presence of atherosclerotic risk factors in the CAD-ACS group; thus, a type II error secondary to low sample sizes could not be excluded for these comparisons. Inflammatory biomarkers were not limited to the ones that were studied in the present work; thus, potential differences in other biomarkers could not be excluded. Because the number of variables evaluated in the present study was severely limited, it could not be assumed that NA-SCAD-ACS or CAD-ACS could not be excluded using other demographic, clinical, laboratory or imaging parameters. Changes in the levels of inflammatory markers during an acute coronary event are not only caused by local reactions within the arterial wall but are also a consequence of the ischemia of the myocardium. Thus, present findings should not necessarily reflect local inflammatory reactions within the coronary vessels, but should be interpreted as changes in the systemic inflammatory biomarkers secondary to all causes of inflammation in ACS.

\section{CONCLUSION}

Similar to the patients with an acute coronary event secondary to coronary atherothrombosis, patients with NA-SCAD demonstrate a systemic inflammatory response during ACS. The degree of inflammation is at least comparable to CAD-ACS and could be even higher in NA-SCAD-ACS, yet the present study could not definitively prove this last assertion. We did not find any evidence for hypereosinophilia despite previous evidence for periarterial eosinophilic infiltration, which suggests that coronary infiltration could be an isolated phenomenon rather than being part of a multiorgan involvement syndrome. In-hospital outcomes in NA-SCAD-ACS patients were not different from those in CAD-ACS patients, although the majority of NA-SCAD-ACS pa- tients were followed up conservatively. As the number of biomarkers tested in the present study was limited due to the retrospective nature of the study, further research on this subject could shed more light on the role of systemic inflammation in the pathophysiology of NA-SCAD.

Conflict of Interest: No conflict of interest was declared by the authors.

Financial Disclosure: The authors declared that this study has received no financial support.

Authorship Contributions: Concept - T.B.; Design - A.N.C., Y.C., T.S.G.; Supervision - M.B.K.; Materials - T.O.; Data collection \&/or processing - A.O.U., V.O.T.; Analysis and/or interpretation - B.G.; Writing - Y.C., T.S.G.; Critical review - O.B.

\section{REFERENCES}

1. Isner JM, Donaldson RF, Fortin AH, Tischler A, Clarke RH. Attenuation of the media of coronary arteries in advanced atherosclerosis. Am J Cardiol 1986;58:937-9. [CrossRef]

2. Aqel RA, Zoghbi GJ, Iskandrian A. Spontaneous coronary artery dissection, aneurysms, and pseudoaneurysms: a review. Echocardiography 2004;21:175-82. [CrossRef]

3. Saw J, Aymong E, Sedlak T, Buller CE, Starovoytov A, Ricci D, et al. Spontaneous coronary artery dissection: association with predisposing arteriopathies and precipitating stressors and cardiovascular outcomes. Circ Cardiovasc Interv 2014;7:645-55. [CrossRef]

4. Robinowitz M, Virmani R, McAllister HA. Spontaneous coronary artery dissection and eosinophilic inflammation: a cause and effect relationship? Am J Med 1982;72:923-8. [CrossRef]

5. Bateman AC, Gallagher PJ, Vincenti AC. Sudden death from coronary artery dissection. J Clin Pathol 1995;48:781-4. [CrossRef]

6. Kajihara H, Tachiyama Y, Hirose T, Takada A, Saito K, Murai T, et al. Eosinophilic coronary periarteritis (vasospastic angina and sudden death), a new type of coronary arteritis: report of seven autopsy cases and a review of the literature. Virchows Arch 2013;462:239-48.

7. Kobayashi M, Cohle SD. A case of sudden cardiac death due to eosinophilic coronary periarteritis: possible significance of coexisting atherosclerosis and eosinophilic inflammation of the esophagus. Cardiovasc Pathol 2016;25:67-71. [CrossRef]

8. Crea F, Liuzzo G. Pathogenesis of Acute Coronary Syndromes. J Am Coll Cardiol 2013;61:1-11. [CrossRef]

9. Libby P. Inflammation in atherosclerosis. Nature 2002;420:868-74.

10. Berk BC, Weintraub WS, Alexander RW. Elevation of C-reactive protein in "active" coronary artery disease. Am J Cardiol 1990;65:168-72.

11. Tomoda H, Aoki N. Prognostic value of $C$-reactive protein levels within six hours after the onset of acute myocardial infarction. Am Heart J 2000;140:324-8. [CrossRef]

12. Liuzzo G, Biasucci LM, Gallimore JR, Grillo RL, Rebuzzi AG, Pepys $\mathrm{MB}$, et al. The prognostic value of $\mathrm{C}$-reactive protein and serum amyloid a protein in severe unstable angina. N Engl J Med 1994;331:41724. [CrossRef]

13. Liuzzo G, Biasucci LM, Gallimore JR, Caligiuri G, Buffon A, Rebuzzi AG, et al. Enhanced inflammatory response in patients with preinfarction unstable angina. J Am Coll Cardiol 1999;34:1696-703. [CrossRef]

14. Biasucci LM, Vitelli A, Liuzzo G, Altamura S, Caligiuri G, Monaco 
C, et al. Elevated levels of interleukin- 6 in unstable angina. Circulation 1996;94:874-7. [CrossRef]

15. Barron HV, Harr SD, Radford MJ, Wang Y, Krumholz HM. The association between white blood cell count and acute myocardial infarction mortality in patients $>$ or $=65$ years of age: findings from the cooperative cardiovascular project. J Am Coll Cardiol 2001;38:1654-61.

16. Cannon CP, McCabe CH, Wilcox RG, Bentley JH, Braunwald E. Association of white blood cell count with increased mortality in acute myocardial infarction and unstable angina pectoris. OPUS-TIMI 16 Investigators. Am J Cardiol 2001;87:636-9. [CrossRef]

17. Yen MH, Bhatt D, Chew DP, Harrington RA, Newby LK, Ardissino $\mathrm{D}$, et al. Association between admission white blood cell count and oneyear mortality in patients with acute coronary syndromes. Am J Med 2003;115:318-21. [CrossRef]

18. Grau AJ, Boddy AW, Dukovic DA, Buggle F, Lichy C, Brandt T, et al; CAPRIE Investigators. Leukocyte count as an independent predictor of recurrent ischemic events. Stroke 2004;35:1147-52. [CrossRef]

19. Toss H, Lindahl B, Siegbahn A, Wallentin L. Prognostic influence of increased fibrinogen and C-reactive protein levels in unstable coronary artery disease. FRISC Study Group. Fragmin during Instability in Coronary Artery Disease. Circulation 1997;96:4204-10. [CrossRef]

20. Sawant AC, Adhikari P, Narra SR, Srivatsa SS, Mills PK, Srivatsa SS. Neutrophil to lymphocyte ratio predicts short-and long-term mortality following revascularization therapy for ST elevation myocardial infarction. Cardiol J 2014;21:500-8. [CrossRef]

21. Park JJ, Jang HJ, Oh IY, Yoon CH, Suh JW, Cho YS, et al. Prognostic value of neutrophil to lymphocyte ratio in patients presenting with ST-elevation myocardial infarction undergoing primary percutaneous coronary intervention. Am J Cardiol 2013;111:636-42. [CrossRef]

22. van der Wal AC, Becker AE, van der Loos CM, Das PK. Site of intimal rupture or erosion of thrombosed coronary atherosclerotic plaques is characterized by an inflammatory process irrespective of the dominant plaque morphology. Circulation 1994;89:36-44. [CrossRef]

23. Isselbacher EM. Thoracic and abdominal aortic aneurysms. Thoracic and abdominal aortic aneurysms. Circulation 2005;111:816-28.
24. He R, Guo DC, Estrera AL, Safi HJ, Huynh TT, Yin Z, et al. Characterization of the inflammatory and apoptotic cells in the aortas of patients with ascending thoracic aortic aneurysms and dissections. J Thorac Cardiovasc Surg 2006;131:671-8. [CrossRef]

25. Weis-Müller BT, Modlich O, Drobinskaya I, Unay D, Huber R, Bojar $\mathrm{H}$, et al. Gene expression in acute Stanford type A dissection: a comparative microarray study. J Transl Med 2006;4:29. [CrossRef]

26. Sakalihasan N, Limet R, Defawe OD. Abdominal aortic aneurysm. Lancet 2005;365:1577-89. [CrossRef]

27. Koller PT, Cliffe CM, Ridley DJ. Immunosuppressive Therapy for Peripartum-Type Spontaneous Coronary Artery Dissection: Case Report and Review. Clin Cardiol 1998;21:40-6. [CrossRef]

28. Biswas M, Sethi A, Voyce SJ. Spontaneous Coronary Artery Dissection: case report and review of literature. Heart Views 2012;13:14954. [CrossRef]

29. Dowling GP, Buja LM. Spontaneous coronary artery dissection occurs with and without periadventitial inflammation. Arch Pathol Lab Med 1987;111:470-2.

30. Kajihara H, Kato Y, Takanashi A, Nakagawa H, Tahara E, Otsuki T, et al. Periarteritis of coronary arteries with severe eosinophilic infiltration. A new pathologic entity (eosinophilic periarteritis)? Path Res Pract 1989;184:46-52. [CrossRef]

31. Alfonso F, Paulo M, Lennie V, Dutary J, Bernardo E, Quevedo PJ, et al. Spontaneous coronary artery dissection: long-term follow-up of a large series of patients prospectively managed with a "conservative" therapeutic strategy. JACC Cardiovasc Interv 2012;5:1062-70. [CrossRef]

32. Ng VG, Lansky AJ, Meller S, Witzenbichler B, Guagliumi G, Peruga JZ, et al. The prognostic importance of left ventricular function in patients with ST-segment elevation myocardial infarction: the HORIZONSAMI trial. Eur Heart J Acute Cardiovasc Care 2014;3:67-77. [CrossRef]

33. Tweet MS, Hayes SN, Pitta SR, Simari RD, Lerman A, Lennon RJ, et al. Clinical features, management, and prognosis of spontaneous coronary artery dissection. Circulation. 2012;126:579-88. [CrossRef] 